\title{
PERFIL LIPÍDICO E GLICÊMICO DE RATOS TREINADOS EM EXERCÍCIO AERÓBIO OU ANAERÓBIO E SUPLEMENTADOS COM MALTODEXTRINA
}

\section{MS. CÁTIA FERNANDES LEITE}

Mestre em Educação Física pela Escola Superior de Educação Física da Universidade Federal de Pelotas (ESEF/UFPel), Pesquisadora do Laboratório de Bioquímica e Fisiologia do Exercício (LABFex/

ESEF/UFPel) (Pelotas - Rio Grande do Sul - Brasil)

E-mail: catialeite@yahoo.com.br

\section{DRA. CLÁUDIA PINHO HARTLEBEN}

Professora Adjunta do Centro de Desenvolvimento Tecnológico, Núcleo Biotecnologia da Universidade Federal de Pelotas (UFPel), Doutorado em Biotecnologia pela Universidade Federal de Pelotas (UFPel) (Pelotas - Rio Grande do Sul - Brasil)

E-mail: clauhart@terra.com.br

\section{GRAD. CAROLINE SOARES MAGALHÃES}

Graduada em Educação Física pela Escola Superior de Educação Física da Universidade Federal de Pelotas (ESEF/UFPel), Professora de educação física da Escola Estadual Rachel Melo (Pelotas - Rio Grande do Sul - Brasil)

E-mail: karolzinhamagah@hotmail.com

\section{DR. AIRTON JOSÉ ROMBALDI}

Professor Associado da Escola Superior de Educação Física da Universidade Federal de Pelotas (ESEF/ UFPel), Pós-Doutorado em Epidemiologia pela Universidade Federal de Pelotas (UFPel), Membro do Grupo de Estudos em Epidemiologia da Atividade Física (GEEAF/ESEF/UFPel), Coordenador do Laboratório de Bioquímica e Fisiologia do Exercício (LABFex/ESEF/UFPel)

(Pelotas - Rio Grande do Sul - Brasil)

E-mail: rombaldi@ufpel.tche.br

\section{RESUMO}

Objetivo: verificar as alterações no perfil lipídico e glicêmico de ratos treinados, suplementados com maltodextrina. Materiais e Métodos: o protocolo de treinamento consistiu de 8 semanas de natação em padrão contínuo $\left(60 \mathrm{~min}\right.$. dia ${ }^{-1}$ ) ou intermitente (2 períodos de $30 \mathrm{~min}$, com intervalo de $10 \mathrm{~min}$ ), com sobrecargas correspondentes a 5\% e $10 \%$ do peso corporal, respectivamente. Durante 37 dias os animais foram suplementados com uma dose diária de 0,48g. $\mathrm{kg}^{\prime}$ de maltodextrina dissolvida em água ou receberam água pura. Resultados: o exercício aeróbio ocasionou aumento significativo no nível glicêmico. Os exercícios e a maltodextrina não causaram alterações no perfil lipídico. Conclusão: o exercício aeróbio proporcionou elevação no nível glicêmico, não causando hipoglicemia.

PALAVRAS-CHAVE: Lipídeos; glicemia; condicionamento; maltodextrina. 
A síndrome metabólica é uma desordem multigenética que abrange várias anormalidades, tais como, obesidade visceral, resistência a insulina, hipertensão arterial, dislipidemia e alteração no controle glicêmico (HARAM et al., 2009). O risco de doença coronária é maior em pessoas sedentárias do que naquelas que são ativas fisicamente, além disso, a inatividade física pode contribuir para hipertensão arterial e baixos níveis de lipoproteína de alta densidade (HDL colesterol) e hipertrigliceridemia, fatores estes associados com doença aterosclerótica (GUERRA et al., 2007).

A baixa capacidade aeróbica pode ser um fator comum que conduz a doença metabólica e cardiovascular (HARAM et al., 2009). Em contraste, a prática de exercícios pode elevar os níveis de aptidão aeróbia e melhorar a capacidade de oxidação dos lipídios (GUERRA et al., 2007), assim como os recursos ergogênicos são capazes de aprimorar a capacidade física ou o desempenho atlético (GUTTIERRES et al., 2009), provavelmente sem afetar o perfil lipídico e glicêmico. O exercício também parece promover efeitos benéficos sobre os lipídios plasmáticos, apesar da influência genética, principalmente em relação aos níveis de HDL colesterol (ZANELLA et al., 20I I).

A relativa oxidação das gorduras e dos carboidratos durante o exercício poderá variar enormemente, mas dependerá muito da intensidade do exercício empregada (VAN LOON et al., 200 I). Os ácidos graxos livres na circulação aumentam durante o exercício devido à mobilização dos estoques de lipídios, ao mesmo tempo em que os estoques de glicogênio diminuem (ILHAN et al., 2004), mas exatamente como o exercício exerce suas ações benéficas prevenindo o risco de doenças cardiovasculares é desconhecido (MEISSNER et al., 20 I 0). De fato, os mecanismos que estão envolvidos por trás de como os exercícios induzem melhoras no perfil lipídico não tem sido definido (MEISSNER et al., 20 I0). Neste sentido, o objetivo deste estudo foi verificar as alterações no perfil lipídico e glicêmico de ratos sedentários, submetidos a exercício aeróbio ou anaeróbio, suplementados com maltodextrina ou que receberam água pura.

\section{MATERIAL E MÉTODOS}

\section{ANIMAIS}

Foram utilizados 69 ratos machos da linhagem Wistar com 60 dias e pesando no início do experimento entre 199-409 gramas. Os animais provenientes do Biotério da Universidade Federal de Pelotas (UFPel) foram alimentados com ração balanceada padrão (Nuvilab ${ }^{\circledR} \mathrm{CRI}$ ), água "ad libitum" e distribuídos em gaiolas coletivas. A temperatura ambiente foi controlada entre $21-25^{\circ} \mathrm{C}$ e fotoperíodo de I $2 \mathrm{~h}$ claro e $12 \mathrm{~h}$ escuro. 


\section{GRUPOS EXPERIMENTAIS}

Os animais foram transferidos para o Laboratório de Bioquímica e Fisiologia do Exercício da UFPel (LABFex/UFPel), pesados e distribuídos, aleatoriamente, em seis grupos: sedentários não suplementados $(n=12)$ e suplementados $(n=12)$; treinados em Estado Estável Máximo de Lactato (EEML) não suplementados $(n=\mid$ I) e suplementados $(n=\mid$ I); treinados em alta intensidade não suplementados $(n=\mid 2)$ e suplementados $(n=||)$.

\section{PROTOCOLO DE TREINAMENTO}

O período de treinamento foi de dez semanas, sendo as duas primeiras de adaptação ao meio líquido (cinco vezes por semana) com sobrecargas progressivas e em tanque coletivo, cilíndrico ( $90 \mathrm{~cm}$ de diâmetro $\times 100 \mathrm{~cm}$ de profundidade), com água a temperatura de $30 \pm 1^{\circ} \mathrm{C}$. As oito semanas subsequentes foram de exercícios de natação, cinco dias consecutivos por semana e 60min por sessão para o exercício de forma contínua ou 70min por sessão para o exercício de forma intermitente (2 períodos de 30min, com 10 min de intervalo, sendo a duração do exercício e do repouso de I5seg) (Figura I).

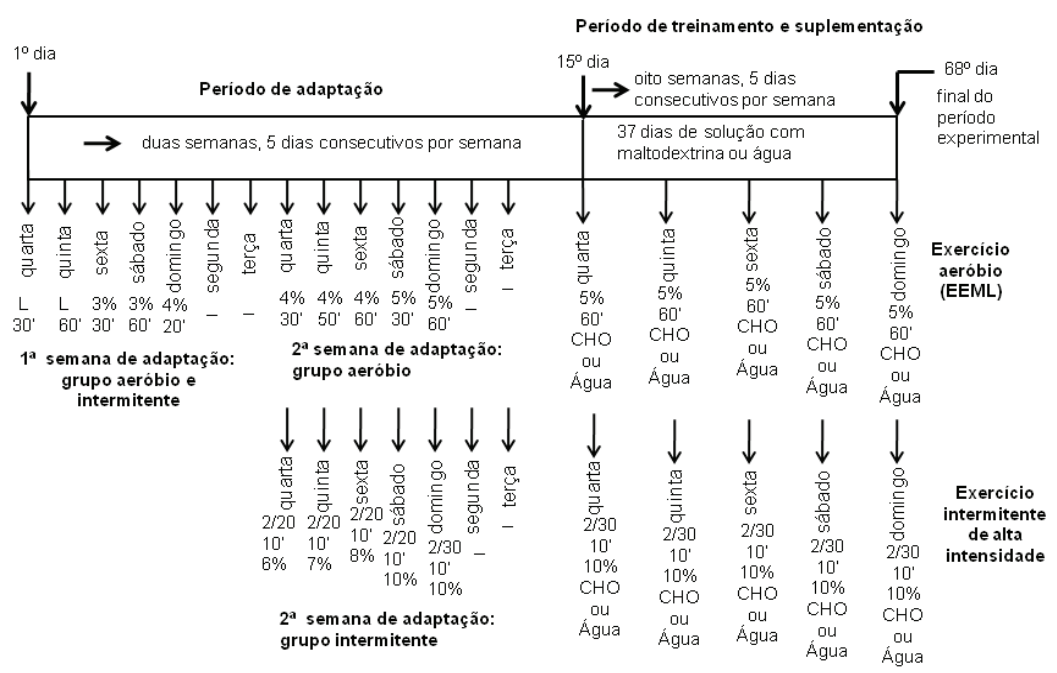

Figura l. Resumo do período de adaptação, treinamento e suplementação. Foram sacrificados quatro animais por grupo, com intervalo de uma semana entre os sacrifícios; L. livre de sobrecarga _: folga 2/20: dois períodos de 20 min com intervalo de $10 \mathrm{~min}$ 2/30: dois períodos de 30min com intervalo de 10 min. $\mathrm{CHO}$ : suplementação com maltodestrina. 
O exercício aeróbio, de padrão contínuo e em carga de EEML foi realizado pelos roedores sem interrupção, até os animais completarem uma hora de treinamento. A sobrecarga utilizada foi a correspondente a $5 \%$ do peso corporal de cada roedor, pois conforme Gobatto et al. (200I), ratos desta linhagem e que foram submetidos a exercício de natação para determinação do EEML (ou como alguns autores o preferem chamar Máxima Fase Estável de Lactato), obtiveram este limiar em 5,5mmol/L de lactato sanguíneo com sobrecargas de 5 e $6 \%$ do peso corporal. A partir do resultado deste estudo, adotou-se a sobrecarga de $5 \%$ do peso corporal para a carga correspondente ao EEML.

As sessões de exercícios de natação de padrão intermitente foram caracterizadas por 70min de duração diária, realizados da seguinte maneira: os animais (em grupos de três e no máximo quatro roedores) eram colocados dentro de um cilindro $(80 \mathrm{~cm}$ de diâmetro $\times 100 \mathrm{~cm}$ de profundidade) $\mathrm{com}$ fundo vazado. Este cilindro era colocado dentro do tanque para que os animais efetuassem I $5 \mathrm{seg}$ de exercício de natação, após este período o cilindro era erguido por um fio de aço para que os animais realizassem I 5 seg de repouso. Este processo, de 15 seg de exercício e 15 seg de repouso, foi executado pelos roedores durante $30 \mathrm{~min}$ (primeiro período). Logo após, os animais realizaram um intervalo de descanso passivo, com IOmin de duração, e depois deste tempo começaram o segundo período de 30min de exercício de natação de padrão intermitente, exatamente como aconteceu no primeiro período. A sobrecarga utilizada foi a correspondente a $10 \%$ do peso corporal, por ser considerada carga de treinamento de alta intensidade e acima do EEML (GOBATTO et al., 200 I).

O peso corporal dos animais foi monitorado todas as segundas-feiras e feita a correção da sobrecarga a partir da alteração no peso. O experimento foi realizado no ciclo claro (GOBATTO et al., 200I), durante o período noturno (ARAÚjO et al., 20 I 0), entre as I 8h00min e 6h00min. Optou-se por aplicar os protocolos de treinamento neste horário para poder dividir os animais em pequenos subgrupos de natação (de três a no máximo quatro roedores por subgrupos) e assim possibilitar menores riscos de perdas de animais por afogamento, e também para assegurar que os animais treinassem sem a interferência de outro roedor. $\bigcirc$ laboratório foi adaptado para obedecer ao ciclo claro/escuro (I $2 \mathrm{~h} / \mathrm{I} 2 \mathrm{~h}$ ).

Os animais dos grupos sedentários foram colocados em tanque com água rasa, em profundidade de $10 \mathrm{~cm}$ (banho de imersão) e temperatura de $30 \pm 1{ }^{\circ} \mathrm{C}$, por $15 \mathrm{~min}$, 5 dias consecutivos por semana, e foram usados como controles. Após cada sessão de treinamento de natação, os roedores foram secos e colocados em ambiente com temperatura entre 21 e $25^{\circ} \mathrm{C}$ para evitar complicações fisiológicas provenientes do frio e da umidade. No último dia do experimento, os animais dos 
grupos treinados em alta intensidade não suplementados e suplementados nadaram até a exaustão. A exaustão foi determinada quando os animais permaneceram submersos por um período superior a 30 segundos (ROMBALDI, 1996). O exercício até a exaustão foi realizado com o objetivo de verificar o efeito do exercício anaeróbio na exaustão sobre as variáveis dependentes deste estudo.

\section{PROTOCOLO DE SUPLEMENTAÇÃO}

Os animais suplementados dos grupos sedentário, treinado em EEML e treinado em alta intensidade foram suplementados através de tubo gástrico ("gavage") com solução carboidratada líquida a $12 \%(\mathrm{~m} / \mathrm{v})$ de maltodextrina dissolvida em água destilada (ROMBALDI, 1996). A dose de carboidrato administrada foi de $0,48 g . \mathrm{Kg}^{\prime}$ de peso, em um volume de $1 \mathrm{~mL}$ para $250 \mathrm{~g}$ de peso animal, e a cada $5 \mathrm{~g}$ de peso superior ou inferior ao peso corporal base, o volume aumentou ou diminuiu em $0,02 \mathrm{~mL}$. Os animais não suplementados dos grupos sedentário, treinado em EEML e treinado em alta intensidade receberam somente água pura, utilizando-se a mesma técnica dos grupos suplementados. Os ratos foram suplementados cinco vezes por semana, durante o período de treinamento, por 37 dias. As soluções foram administradas aos animais dos grupos treinados após os roedores serem submetidos a aquecimento prévio de natação por 2 min.

\section{AMOSTRAS SANGUÍNEAS E ANÁLISES}

O sacrifício dos animais ocorreu no último dia de treinamento, imediatamente após as sessões de exercício aeróbio, ou exercício de exaustão, ou após uma hora de repouso depois de efetuadas as administrações da solução com maltodextrina ou de água pura para os animais dos grupos sedentários, sendo coletadas amostras sanguíneas sem anticoagulante. $\bigcirc$ soro foi separado por centrifugação a 3000rpm por $10 \mathrm{~min}$. Alíquotas deste material recém obtido foram armazenadas à $-20^{\circ} \mathrm{C}$ para posterior análise das variáveis bioquímicas. As análises de glicose sérica, colesterol total, HDL colesterol, LDL colesterol e triglicérides foram feitas por espectrofotometria e seguiram as determinações dos kits comerciais da marca Labtest (Lagoa Santa/MG/Brasil).

\section{ANÁLISE DE DADOS}

A análise estatística foi conduzida no pacote estatístico STATISTICA para Windows, versão 8, da Statsoft. Quando as variáveis seguiram a curva normal, foi empregada a análise de variância fatorial para a comparação entre as médias. Para 
as variáveis que apresentaram comportamento não paramétrico, se utilizou o teste Kruskal-Wallis. Os valores foram expressos como médias e desvios padrões, sendo adotado o nível de significância de $p<0,05$.

\section{PROCEDIMENTOS ÉTICOS}

Este estudo foi realizado de acordo com as normas sugeridas pelo Colégio Brasileiro de Experimentação Animal (COBEA) e aprovado pela Comissão de Ética em Experimentação Animal (CEEA) da UFPel (Processo número 5873/2009).

\section{RESULTADOS E DISCUSSÃO}

No início do período experimental, os pesos corporais dos animais dos grupos experimentais, aeróbio e anaeróbio que receberam água foram significativamente maiores do que o peso corporal dos animais do grupo experimental sedentário que receberam água pura, ( $p<0,005$ e $p<0,02$, respectivamente) (TABELA I). Como a distribuição dos animais nos grupos experimentais foi realizada de maneira aleatória, não foi possível fazer o emparelhamento dos grupos por peso corporal inicial. Esta diferença de menor peso corporal inicial no grupo de animais sedentários e que receberam água pura, não compromete a interpretação dos resultados deste estudo, uma vez que, após dez semanas de treinamento os pesos corporais finais dos animais nos diferentes grupos experimentais não foram divergentes $(p>0,05)$ (TABELA I), ou seja, todos os animais tiveram a mesma chance de aumentarem seus pesos corporais.

Tabela I. Peso corporal de ratos Wistar sedentários, treinados, suplementados com maltodextrina ou que receberam água pura

\begin{tabular}{lllllll}
\hline \multirow{2}{*}{ Peso corporal } & Sed & \multicolumn{3}{c}{ Aeróbio } & Anaeróbio & \\
\cline { 2 - 6 } & Água $(n=12)$ & $\mathrm{CHO}(\mathrm{n}=12)$ & Água $(\mathrm{n}=11)$ & $\mathrm{CHO}(\mathrm{n}=11)$ & Água $(\mathrm{n}=12)$ & $\mathrm{CHO}(\mathrm{n}=11)$ \\
\hline & $292,1 \pm 23,3$ & $320,6 \pm 62,4$ & $347,9 \pm 13,3^{*}$ & $346,3 \pm 19,4$ & $352,5 \pm 15,6^{\#}$ & $323,5 \pm 36,8$ \\
Inicial (gramas) & & & & & & \\
Final (gramas) & $409,4 \pm 41,8$ & $440,6 \pm 72,9$ & $402,1 \pm 39,5$ & $411,5 \pm 24,7$ & $425,5 \pm 25,8$ & $399,8 \pm 49,8$ \\
\hline
\end{tabular}

Os valores estão expressos como médias e desvio padrão. "Água" corresponde aos animais que receberam água pura. "CHO" corresponde aos animais suplementados com maltodextrina. Sed: animais sedentários. Aeróbio: animais treinados em exercício aeróbio contínuo sob carga de Estado Estável Máximo de Lactato. Anaeróbio: animais treinados em exercício anaeróbio de alta intensidade. " $p<0,005$ versus Sed água. ${ }^{*} p<0,02$ versus Sed água. Teste estatístico utilizado foi o Kruskal Wallis. 
Os animais treinados em exercício aeróbio sob carga de EEML e que receberam água pura apresentaram um aumento significativo na concentração de glicose sérica, comparado ao grupo de animais sedentários e que receberam água pura. Não houve diferença significativa nos valores glicêmicos entre o grupo de ratos treinados em exercício anaeróbio de alta intensidade e que receberam água pura comparado com o grupo de animais sedentários que receberam água pura. Também não houve efeito significativo da suplementação com maltodextrina na concentração de glicose sérica entre os diferentes grupos experimentais do presente estudo (TABELA 2).

treinamento físico de padrão aeróbio contínuo sob carga de EEML ou de padrão anaeróbio de alta intensidade e a utilização de suplementação carboidratada associada ao uso de água pura não ocasionaram diferenças estatísticas significativas ( $p>0,05)$ no perfil lipídico destes animais, comparado aos ratos dos grupos controles (sedentários que receberam água pura ou sedentários suplementados com maltodextrina) (TABELA 2).

Tabela 2. Efeitos do treinamento aeróbio e anaeróbio sobre parâmetros bioquímicos de ratos Wistar suplementados com maltodextrina ou que receberam água pura

\begin{tabular}{|c|c|c|c|c|c|c|}
\hline \multirow[b]{2}{*}{ Parâmetros bioquímicos } & \multicolumn{2}{|l|}{ Sed } & \multicolumn{2}{|l|}{ Aeróbio } & \multicolumn{2}{|l|}{ Anaeróbio } \\
\hline & Água $(n=12)$ & $\mathrm{CHO}(\mathrm{n}=12)$ & Água $(n=\mid 1)$ & $\mathrm{CHO}(n=11)$ & Água $(n=12)$ & $\mathrm{CHO}(\mathrm{n}=11)$ \\
\hline Glicemia, (mg/dL) & $116,3 \pm 24,6$ & $130,5 \pm 15,5$ & $195,9 \pm 81,9 *$ & $214,5 \pm 92,7$ & $|29| \pm 29,4$, & $134,3 \pm 50,4$ \\
\hline Colesterol total, (mg/dL) & $140,3 \pm 33,0$ & $150,0 \pm 35,8$ & $|4|, 3 \pm 40,3$ & $135,8 \pm 29,1$ & $132,0 \pm 30,9$ & $|3|, 4 \pm 23,5$ \\
\hline HDL colesterol, (mg/dL) & $50,7 \pm 14,6$ & $41,4 \pm 10,5$ & $38,1 \pm 12,1$ & $43,5 \pm 12,3$ & $44,8 \pm 17,0$ & $46,1 \pm 23,4$ \\
\hline LDL colesterol, (mg/dL) & $90,4 \pm 43,2$ & $108,6 \pm 35,6$ & $103,2 \pm 48,0$ & $92,4 \pm 36,0$ & $87,2 \pm 45,6$ & $85,3 \pm 37,8$ \\
\hline Triglicérides, (mg/dL) & $119,8 \pm 39,7$ & $|2|, 3 \pm 4 \mid, 0$ & $109,7 \pm 36,1$ & $|26| \pm 53,7$, & $113,6 \pm 25,7$ & $107,9 \pm 26,8$ \\
\hline
\end{tabular}

Os valores estão expressos como médias e desvios padrões. "Água" corresponde aos animais que receberam água pura. "CHO" corresponde aos animais suplementados com maltodextrina. Sed: animais sedentários. Aeróbio: animais treinados em exercício aeróbio sob carga de Estado Estável Máximo de Lactato. Anaeróbio: animais treinados em exercício anaeróbio de alta intensidade. " $p<0,02$ versus Sed água. Variável glicemia foi usado o teste estatístico Kruskal Wallis. O teste estatístico ANOVA fatorial foi utilizado para as demais variáveis.

Animais treinados em exercício aeróbio e anaeróbio que receberam água pura apresentaram maiores valores de pesos corporais no início do período experimental. No final de dez semanas os animais dos diferentes grupos experimentais demonstraram não haver diferenças nos pesos corporais finais. Isto indica que a suplementação com maltodextrina não acarretou aumentos divergentes no ganho de peso corporal. $\bigcirc$ aumento na oferta de carboidratos poderá não acarretar elevações no ganho de peso corporal. Conforme Moura et al. (2008), uma dieta rica em frutose administrada a ratos comparada com uma dieta balanceada não 
ocasionou diferenças significativas nos ganhos de pesos corporais. Em humanos, as utilizações de maltodextrina associada a outras fontes nutricionais suplementares não ocasionaram diferenças nos pesos corporais (TREVISAN; SOUZA; MARUCCI, 2010; COELHO et al., 20I0).

Embora a prática de exercícios físicos possa ser benéfica para a saúde, ainda não há um consenso a respeito de qual o melhor tipo de exercício ou intensidade que deveria ser praticado (ZANELLA et al., 20 I I). No presente estudo, tanto o exercício aeróbio contínuo quanto o anaeróbio de alta intensidade não ocasionaram alterações no perfil lipídico dos animais, porém os ratos que receberam água pura e realizaram exercício aeróbio demonstraram haver um aumento na concentração de glicose sérica com este padrão de treinamento. Esta elevação no nível glicêmico era esperada, pois conforme Mamus et al. (2006), o aumento da glicemia associada com outros fatores estão envolvidos com o atraso no começo da fadiga.

Alguns estudos demonstraram haver respostas glicêmicas e lipídicas diferentes do presente trabalho. Em um estudo realizado com jogadores de futebol profissionais comparado a um grupo de homens escolhidos na população geral e que não realizaram exercícios físicos regulares, observou-se que a glicemia foi maior no grupo controle comparado ao grupo de jogadores de futebol, enquanto os níveis de colesterol total, triglicérides, HDL colesterol e LDL colesterol não diferiram entre os grupos (ZANELLA et al., 20 I I). Em outro estudo, com um grupo de trabalhadores japoneses que completaram três meses de um programa de exercício aeróbio associado com um de resistência muscular, foram demonstradas diminuições significativas nos níveis de triglicérides, colesterol total, LDL colesterol, aumento significativo nas concentrações de HDL colesterol e nenhuma alteração no perfil glicêmico destes trabalhadores do pré-exercício comparado com três meses de treinamento (GUO et al., 20 I I). Meissner et al. (20 I0) realizaram um estudo com ratos que correram em roda para corrida voluntária durante duas semanas, e observaram que o treinamento aeróbio não ocasionou alterações nas concentrações de triglicérides, porém houve uma diminuição significativa nos níveis plasmáticos de colesterol total dos ratos exercitados comparados aos animais mantidos sedentários.

Do ponto de vista de reposição energética, uma solução esportiva isotônica contendo maltodextrina pode conter cinco vezes mais calorias do que uma suplementação esportiva isotônica contendo glicose (INAGAKl et al., 20 I I). Os benefícios da suplementação com maltodextrina podem ser explicados principalmente pela manutenção dos altos níveis de glicose sanguínea, evitando provavelmente, um decréscimo no desempenho associado à hipoglicemia (MAMUS et al., 2006). No presente estudo a suplementação com maltodextrina não ocasionou alterações no nível glicêmico e perfil lipídico dos diferentes grupos experimentais. 
Suplementações contendo diferentes fontes de carboidratos apresentaram diversos efeitos sobre a glicemia e perfil lipídico. Um estudo desenvolvido com ratos Wistar que consumiram uma dieta balanceada ou uma dieta rica em frutose, observou-se que a concentração de triglicérides foi maior no grupo que se alimentou com a dieta rica em frutose, entretanto, os níveis glicêmico, colesterol total, HDL colesterol, LDL colesterol não foram diferentes entre os animais alimentados com dieta balanceada comparado aos animais alimentados com dieta rica em frutose (MOURA et al., 2008).

Em humanos observaram-se os seguintes resultados: em dois grupos de indivíduos ativos submetidos a exercícios de força de membros inferiores e suplementados com uma solução esportiva contendo glicose ou solução contendo glicose e frutose, identificou-se que os valores de triglicérides foram maiores quando os sujeitos receberam a solução glicose e frutose do que quando foram suplementados somente com glicose durante o exercício. Também se observaram elevações na glicemia em 30 min de exercício comparado aos níveis de repouso, quando se usou a solução contendo glicose (SÁ; FERNÁNDEZ; SILVA-GRIGOLETTO, 20 I0). Homens não atletas, saudáveis, realizaram três testes submáximos no segundo limiar ventilatório até chegar à exaustão ou até completarem uma hora de exercício. Os voluntários, 30min antes do início dos testes, ingeriram ou uma solução placebo (sem carboidratos), ou solução contendo maltodextrina, ou solução contendo glicose. Os níveis glicêmicos em 15 min e 30min de exercício não apresentaram diferenças significativas entre os três grupos experimentais (SAPATA; FAYH; OLIVEIRA, 2006).

De acordo com o exposto, as limitações do presente estudo referem-se à falta de avaliações dos hormônios insulina e da lipase hormônio sensível para uma interpretação mais apurada da real contribuição dos carboidratos ou lipídios para o gasto energético durante os exercícios. Também a necessidade de avaliações das concentrações de fosfato inorgânico para a contribuição da creatina fosfato na produção de energia durante exercícios de alta intensidade. Estas limitações de forma alguma comprometem os resultados deste estudo e servem como bases para possíveis novas investigações nesta área.

\section{CONCLUSÕES}

A partir dos resultados encontrados, conclui-se que os tipos de exercícios físicos e a utilização de uma solução esportiva contendo maltodextrina não ocasionaram alterações no perfil lipídico; entretanto, o exercício aeróbio proporcionou elevações nos níveis glicêmicos, estabelecendo que este padrão de treinamento de intensidade correspondente ao EEML e com uma hora de duração ainda consegue manter a oferta de glicose para esta demanda energética, sem ocasionar hipoglicemia. 
Lipids and glucose levels of trained rats in aerobic or anaerobic conditions and supplemented with maltodextrin

ABSTRACT: The objective was to verify changes in lipids and glucose levels of trained rats supplemented with maltodextrin. Materials and Methods: The training protocol consisted of 8 weeks of continuous swimming pattern $\left(60 \mathrm{~min}\right.$. dia ${ }^{-1}$ ) or intermittent (2 periods of 30 minutes, with an interval of 10 minutes), with overloads corresponding to $5 \%$ and $10 \%$ of body weight, respectively. For 37 days the animals were supplemented with a daily dose of 0.48 g. $k g-1$ maltodextrin dissolved in water or pure water. Results: Aerobic exercise caused a significant increase in blood glucose levels. The exercises and maltodextrin did not cause changes in lipid profile. Conclusion: Aerobic exercise gave rise in blood glucose levels, without causing hypoglycemia.

KEYWORDS: Lipids; blood glucose; conditioning; maltodextrin.

\section{Los lípidos y los niveles de glucosa de las ratas entrenadas en condiciones aeróbicas o anaeróbicas y se complementa con maltodextrina}

RESUMEN: El objetivo fue comprobar los cambios en los lípidos y los niveles de glucosa de las ratas entrenadas complementados con maltodextrina. Material y Métodos: El protocolo consistió en 8 semanas de patrón de natación continua ( $\left.60 \mathrm{~min}_{\text {. dia }}{ }^{-1}\right)$ o intermitente (2 períodos de $30 \mathrm{~min}$, con un intervalo de $10 \mathrm{~min}$ ), con sobrecargas que corresponden al $5 \%$ y el 10\% del peso corporal. Durante 37 días los animales fueron suplementados con una dosis diaria de 0,48 g. kg- I maltodextrina disuelta en agua o agua pura. Resultados: El ejercicio aeróbico provocó un aumento significativo en los niveles de glucosa en la sangre. Los ejercicios y la maltodextrina no causó cambios en el perfil lipídico. Conclusiones: El ejercicio aeróbico dio lugar en los niveles de glucosa en la sangre, sin causar hipoglucemia.

PALABRAS-CLAVE: Lípidos; glucemia; condicionamiento; maltodextrina.

\section{REFERÊNCIAS}

ARAÚJO, M. B. et al. Treinamento em diferentes intensidades e biomarcadores de estresse oxidativo e do metabolismo glicídico musculoesquelético de ratos. Revista da Educação Físical UEM, Maringá, v. 21 , n. 4, p. 695-707, 2010.

COELHO, C. F. et al. A suplementação de L-carnitina não promove alterações na taxa metabólica de repouso e na utilização dos substratos energéticos em indivíduos ativos. Arquivos Brasileiros de Endocrinologia e Metabologia, São Paulo, v. 54, n. I, p. 37-44, fev. 2010.

GOBATTO, C. A. et al. Maximal lactate steady state in rats submitted to swimming exercise. Comparative Biochemistry and Physiology, Oxford, v. 130, n. I, p. 21-27, 2001.

GUERRA, R. L. F. et al. Effects of 2 or 5 consecutive exercise days on adipocyte area and lipid parameters in Wistar rats. Lipids in Health and Disease, London, v. 6, n. I6, p. I-8, jul. 2007. 
GUO, W. et al. Effects of aerobic exercise on lipid profiles and high molecular weight adiponectin in japanese workers. Internal Medicine, Tokyo, v. 50, n. 5, p. 389-395, mar. 201 I.

GUTTIERRES, A. P. M. et al. Efeito ergogênico de uma bebida esportiva cafeinada sobre a performance em testes de habilidades específicas do futebol. Revista Brasileira de Medicina do Esporte, São Paulo, v. I5, n. 6, p. 450-454, nov./dez. 2009.

HARAM, P. M. et al. Aerobic interval training vs. continuous moderate exercise in the metabolic syndrome of rats artificially selected for low aerobic capacity. Cardiovascular Research, London, v. 81, n. 4, p. 723-732, dec. 2009.

ILHAN, N. et al. Variable effects of exercise intensity on reduced glutathione, thiobarbituric acid reactive substance levels, and glucose concentration. Archives of Medical Research, México, v. 35, n. 4, p. 294-300, jul./aug. 2004.

INAGAKI, K. et al. Rapid rehydration and moderate plasma glucose elevation by fluid containing enzymatically synthesized glycogen. Journal of Nutritional Science Vitaminology, Tokyo, v. 57, n. 2, p. 170-176, 201।.

MAMUS, R. T. et al. Biochemical effects of carbohydrate supplementation in a simulated competition of short terrestrial duathlon. Journal of the International Society Sports Nutrition, London, v. 3, n. 2, p. 6-। I, 2006.

MEISSNER, M. et al. Exercise enhances whole-body cholesterol turnover in mice. Medicine and Science in Sports and Exercise, Hagerstown, v. 42, n. 8, p. I460-1468, aug. 2010.

MOURA, R. F. et al. Capacidade aeróbia de ratos alimentados com dieta rica em frutose. Revista Brasileira de Medicina do Esporte, São Paulo, v. I4, n. 5, p. 422-426, set./out. 2008.

ROMBALDI, A. J. Alguns efeitos bioquímicos da ingestão de carboidrato líquido na realização de trabalho intermitente de alta intensidade em ratos. 1996. 265 f. Tese (Doutorado em Ciências do Movimento Humano) - Centro de Educação Física e Desportos, Universidade Federal de Santa Maria, Santa Maria, 1996.

SÁ, C. A.; FERNÁNDEZ, J. M.; SILVA-GRIGOLETTO, M. E. Respostas metabólicas à suplementação com frutose em exercício de força de membros inferiores. Revista Brasileira de Medicina do Esporte, São Paulo, v. 16, n. 3, p. 176-181, maio/jun 2010.

SAPATA, K. B.; FAYH, A. P. T.; OLIVEIRA, A. R. Efeitos do consumo prévio de carboidratos sobre a resposta glicêmica e desempenho. Revista Brasileira de Medicina do Esporte, São Paulo, v. 12, n. 4, p. 189-194, jul./ago. 2006.

TREVISAN, M. C.; SOUZA, J. M. P.; MARUCCI, M. F. N. Influence of soy protein intake and weight training on the resting energy expenditure of postmenopausal women. Revista da Associação Médica Brasileira, São Paulo, v. 56, n. 5, p. 572-578, 2010. 
VAN LOON, L. J. C. et al. The effects of increasing exercise intensity on muscle fuel utilization in humans. Journal of Physiology, London, v. 536, n. I, p. 295-304, 200 I.

ZANELLA, A. M. et al. Lipid profile, apolipoprotein A-I and oxidative stress in professional footballers, sedentary individuals, and their relatives. Arquivos Brasileiros de Endocrinologia e Metabologia, São Paulo, v. 55, n. 2, p. 121-126, 2011.

Recebido em: 17 ago. 2011

Aprovado em: 2 fev. 2012

Endereço para correspondência:

Airton José Rombaldi

Rua Luiz de Camões, 625

Bairro Três Vendas

Pelotas - RS

CEP: 96055-630 